\title{
THE HIGH ORDER BLOCK RIP CONDITION FOR SIGNAL RECOVERY*
}

\author{
Yaling Li \\ School of Science, Zhejiang University of Science and Technology, Hangzhou, Zhejiang, 310023, China \\ Email: leeyaling@126.com \\ Wengu Chen \\ Institute of Applied Physics and Computational Mathematics, Beijing, 100088, China \\ Email: chenwg@iapcm.ac.cn
}

\begin{abstract}
In this paper, we consider the recovery of block sparse signals, whose nonzero entries appear in blocks (or clusters) rather than spread arbitrarily throughout the signal, from incomplete linear measurements. A high order sufficient condition based on block RIP is obtained to guarantee the stable recovery of all block sparse signals in the presence of noise, and robust recovery when signals are not exactly block sparse via mixed $l_{2} / l_{1}$ minimization. Moreover, a concrete example is established to ensure the condition is sharp. The significance of the results presented in this paper lies in the fact that recovery may be possible under more general conditions by exploiting the block structure of the sparsity pattern instead of the conventional sparsity pattern.
\end{abstract}

Mathematics subject classification: 90C59, 94A12, 94A20.

Key words: Block sparsity, Block restricted isometry property, Compressed sensing, Mixed $l_{2} / l_{1}$ minimization.

\section{Introduction}

Compressed sensing (CS), a new type of sampling theory, is a fast growing field of research. It has attracted considerable interest in a number of fields including applied mathematics, statistics, seismology, signal processing and electrical engineering. Interesting applications include radar system [26,50], coding theory [1,13], DNA microarrays [39], color imaging [33], magnetic resonance imaging [31]. Up to now, there are already many works on CS [3,4,15-17,29,30,40-44]. The key problem in CS is to recover an unknown high-dimensional sparse signal $x \in \mathbb{R}^{N}$ using an efficient algorithm through a sensing matrix $A \in \mathbb{R}^{n \times N}$ and the following linear measurements

$$
y=A x+z
$$

where the observed signal $y \in \mathbb{R}^{n}, n \ll N$ and the vector of measurement errors $z \in \mathbb{R}^{n}$. In general, the solutions to the underdetermined system of linear equations (1.1) are not unique. But now suppose that $x$ is known to be sparse in the sense that it contains only a small number of nonzero entries, which can occur in anywhere in $x$. This premise fundamentally changes the problem such that there is a unique sparse solution under regularity conditions. It is well known the $l_{1}$ minimization approach, a widely used algorithm, is an effective way to recover sparse signals in many settings. One of the most widely used frameworks to depict recovery

\footnotetext{
${ }^{*}$ Received July 7, 2017 / Revised version received September 14, 2017 / Accepted October 10, 2017 /

Published online August 22, 2018 /
} 
ability of $l_{1}$ minimization in CS is the restricted isometry property (RIP) introduced by Candès and Tao [13]. Let $A \in \mathbb{R}^{n \times N}$ be a matrix and $1 \leq k \leq N$ is an integer, the restricted isometry constant (RIC) $\delta_{k}$ of order $k$ is defined as the smallest nonnegative constant that satisfies

$$
\left(1-\delta_{k}\right)\|x\|_{2}^{2} \leq\|A x\|_{2}^{2} \leq\left(1+\delta_{k}\right)\|x\|_{2}^{2},
$$

for all $k$-sparse vectors $x \in \mathbb{R}^{N}$. A vector $x \in \mathbb{R}^{N}$ is $k$-sparse if $|\operatorname{supp}(x)| \leq k$, where $\operatorname{supp}(x)=$ $\left\{i: x_{i} \neq 0\right\}$ is the support of $x$. When $k$ is not an integer, we define $\delta_{k}$ as $\delta_{\lceil k\rceil}$. It has been shown $l_{1}$ minimization can recover a sparse signal with a small or zero error under some appropriate RIC met by the measurement matrix $A[5-12,23,24,37]$. As far as we know, a sharp sufficient condition based on RIP for exact and stable recovery of signals in both noiseless and noisy cases by $l_{1}$ minimization was established by Cai and Zhang [8].

However, in practical examples, there are signals which have a particular sparsity pattern, where the nonzero coefficients appear in some blocks (or clusters). Such signals are referred to as block sparse $[19,20,46]$. In practice, the block sparse structure is very common, such as reconstruction of multi-band signals [35], equalization of sparse communication channels [18] and multiple measurement vector (MMV) model [20,21,36]. Actually, the notion of block sparsity was already introduced in statistics literature and was named the group Lasso estimator $[2,14,27,34,38,48]$. Recently, block sparsity pattern has attracted significant attention. Various efficient methods and explicit recovery guarantees $[19,20,22,25,28,32,45-47]$ have been proposed.

In this paper, our goal is to recover the unknown signal $x$ from linear measurements (1.1). But at the moment, nonzero elements of signal $x$ are occurring in blocks (or clusters) instead of spreading arbitrarily throughout the signal vector. To this end, firstly, we need the concept of block sparsity. In order to emphasize the block structure, similar to $[20,46]$, we view $x$ as a concatenation of blocks over $\mathcal{I}=\left\{d_{1}, d_{2}, \ldots, d_{M}\right\}$. Then $x$ can be expressed as

$$
x=(\underbrace{x_{1}, \ldots, x_{d_{1}}}_{x[1]}, \underbrace{x_{d_{1}+1}, \ldots, x_{d_{1}+d_{2}}}_{x[2]}, \ldots, \underbrace{x_{N-d_{M}+1}, \ldots, x_{N}}_{x[M]})^{T} \in \mathbb{R}^{N},
$$

where $x[i]$ denotes the $i$ th block of $x$ with the length $d_{i}$ and $N=\sum_{i=1}^{M} d_{i}$. A vector $x \in \mathbb{R}^{N}$ is called block $k$-sparse over $\mathcal{I}=\left\{d_{1}, d_{2}, \ldots, d_{M}\right\}$ if the number of nonzero vectors $x[i]$ is at most $k$ for $i \in\{1,2, \ldots, M\}$. Define

$$
\|x\|_{2,0}=\sum_{i=1}^{M} I\left(\|x[i]\|_{2}>0\right),
$$

where $I(\cdot)$ is an indicator function that it equals to 1 if its argument is larger than zero and 0 elsewhere. Then the block $k$-sparse vector over $\mathcal{I}=\left\{d_{1}, d_{2}, \ldots, d_{M}\right\}$ can be cast as $\|x\|_{2,0} \leq k$. If $d_{i}=1$ for all $i$, block sparsity is just the conventional sparsity. Next, one of the efficient methods to recover block sparse signals is mixed $l_{2} / l_{1}$ minimization

$$
\min _{x}\|x\|_{2,1}, \quad\|y-A x\|_{2} \leq \varepsilon
$$

where $\|x\|_{2,1}=\sum_{i=1}^{M}\|x[i]\|_{2}$. Moreover, mixed norm $\|x\|_{2,2}=\left(\sum_{i=1}^{M}\|x[i]\|_{2}^{2}\right)^{1 / 2}$ and $\|x\|_{2, \infty}=$ $\max _{i}\|x[i]\|_{2}$. Note that $\|x\|_{2,2}=\|x\|_{2}$. It is easy to know the mixed norm minimization is a generalization of conventional norm minimization. To ensure uniqueness and stability of solution for the system (1.1) via mixed $l_{2} / l_{1}$ minimization, Eldar and Mishali [20] generalized the notion of standard restricted isometry property to block sparse vectors, and obtained the following concept of block restricted isometry property (block RIP). 
Definition 1.1 (block RIP) Let $A \in \mathbb{R}^{n \times N}$ be a matrix, then $A$ has the $k$ order block restricted isometry property over $\mathcal{I}=\left\{d_{1}, d_{2}, \ldots, d_{M}\right\}$ with nonnegative parameter $\delta_{k \mid \mathcal{I}}$ if

$$
\left(1-\delta_{k \mid \mathcal{I}}\right)\|x\|_{2}^{2} \leq\|A x\|_{2}^{2} \leq\left(1+\delta_{k \mid \mathcal{I}}\right)\|x\|_{2}^{2}
$$

holds for all block $k$-sparse vectors $x \in \mathbb{R}^{N}$ over $\mathcal{I}$. The smallest constant $\delta_{k \mid \mathcal{I}}$ is called block restricted isometry constant (block RIC). When $k$ is not an integer, we define $\delta_{k \mid \mathcal{I}}$ as $\delta_{\lceil k\rceil \mid \mathcal{I}}$.

For simplicity, we use $\delta_{k}$ for the block RIP constant $\delta_{k \mid \mathcal{I}}$ in the remainder of this paper. The block RIP plays a role similar to standard RIP. The block RIP provides recovery guarantee for block sparse signals. For example, Eldar and Mishali [20] proved that if matrix $A$ satisfies block restricted isometry constant (block RIC) $\delta_{2 k}<\sqrt{2}-1$, the mixed $l_{2} / l_{1}$ minimization can recover exactly the block $k$-sparse signals in noiseless case, and can approximate the best block $k$-sparse solution in the presence of noise and mismodeling errors. Furthermore, they illustrated the advantage of block RIP over standard RIP. That is, the probability to satisfy the standard RIP is less than that of satisfying the block RIP. Meanwhile, a specific example is given to account for the advantage. They also experimentally demonstrated the advantage of their algorithm (mixed $l_{2} / l_{1}$ minimization) over standard basis pursuit. This explained the performance advantage of block sparse recovery over standard sparse recovery. Later, Lin and Li [28] improved the bound of block RIC to $\delta_{2 k}<0.4931$, and also gave another one order sufficient condition of recovery based on block RIC $\delta_{k}<0.307$. There are many other recovery guarantees and efficient recovery methods to ensure the recovery of signals with special structure. For example, block coherence [19], strong group sparsity [27], $l_{2} / l_{p}(0<p<1)$ minimization [46], BOMP [25, 47].

In this paper, we investigate the high order block RIP conditions for the exact or stable recovery of signals with block structure from (1.1) via solving mixed $l_{2} / l_{1}$ minimization in noiseless and noisy cases. Using ideas similar to [8], we establish a sufficient condition on $\delta_{t k}$ to ensure the stable or exact recovery of signals with nonzero entries occurring in blocks (or clusters) rather than being arbitrarily spread throughout the signal vector. The key is to generalize the technique of sparse representation of a polytope [8] to the block setting. We show that block RIC $\delta_{t k}<\sqrt{\frac{t-1}{t}}$ for any $t>1$ can ensure exact and stable recovery for all block sparse signals and robust recovery for nearly block sparse signals via mixed $l_{2} / l_{1}$ minimization. Moreover, it is sharp when $t \geq 4 / 3$. A concrete example is given to illustrate the optimality. Actually, our results are a generalization of that of Cai and Zhang [8] in the block setting. When $d_{i}=1$ for $i \in\{1, \ldots, M\}$, our results return to those of Cai and Zhang [8]. The significance of our results lies in the fact that taking advantage of explicit block sparsity has better reconstruction performance than viewing the signals as being standard sparsity, accordingly ignoring the additional structure in the problem.

The rest of the paper is organized as follows. In Section 2, we will introduce some notations and establish some basic lemmas that will be used. The main results and their proofs are given in Section 3. Finally, we summarize this paper in Section 4.

\section{Preliminaries}

Throughout this paper, we adopt the following notations unless otherwise stated. For any $x \in \mathbb{R}^{N}$, we model it over $\mathcal{I}=\left\{d_{1}, d_{2}, \ldots, d_{M}\right\} . x[i]$ denotes the $i$ th block of $x$. Let $\mathbf{0}$ be the zero vector whose dimension may be different. $\Gamma \subset\{1,2, \ldots, M\}$ indicates block indices, $\Gamma^{c}$ is the 
complement of $\Gamma$ in $\{1,2, \ldots, M\} . x[\Gamma] \in \mathbb{R}^{N}$ denotes a vector which equals to $x$ on block indices $\Gamma$ and 0 otherwise. For example, if $\Gamma=\{1,3, M\}$, then $x[\Gamma]=(x[1], \mathbf{0}, x[3], \mathbf{0}, \ldots, \mathbf{0}, x[M])^{T} \in$ $\mathbb{R}^{N}$, and $x[\Gamma][i]$ denotes $i$ th block of $x[\Gamma]$. We denote by $\operatorname{supp}[x]=\left\{i:\|x[i]\|_{2} \neq 0\right\}$ the block support of $x$, and $\mathcal{I}_{0}$ the block indices of the $k$ largest block in $l_{2}$ norm of $x$, i.e., $\|x[i]\|_{2} \geq\|x[j]\|_{2}$ for any $i \in \mathcal{I}_{0}$ and $j \in \mathcal{I}_{0}^{c}$. We also denote $x[\max (k)]$ as $x$ with all but the largest $k$ blocks in $l_{2}$ norm set to zero. From now on, we always take that $h=\widehat{x}-x$, where $\widehat{x}$ is the minimizer of $l_{2} / l_{1}$ minimization problem (1.2) and $x$ is the original signal.

The following lemma provides a key technical tool for the proof of our main result. It is an extension of Lemma 1.1 introduced by Cai and Zhang [8]. We extend sparse representation of a polytope to the block setting.

Lemma 2.1. For a positive number $\alpha$ and a positive integer $k$, define the block polytope $T(\alpha, k) \subset \mathbb{R}^{N}$ by

$$
T(\alpha, k)=\left\{v \in \mathbb{R}^{N}:\|v\|_{2, \infty} \leq \alpha,\|v\|_{2,1} \leq k \alpha\right\} .
$$

For any $v \in \mathbb{R}^{N}$, define the set of block sparse vectors $U(\alpha, k, v) \subset \mathbb{R}^{N}$ by

$$
U(\alpha, k, v)=\left\{u \in \mathbb{R}^{N}: \operatorname{supp}(u) \subseteq \operatorname{supp}(v),\|u\|_{2,0} \leq k,\|u\|_{2,1}=\|v\|_{2,1},\|u\|_{2, \infty} \leq \alpha\right\} .
$$

Then any $v \in T(\alpha, k)$ can be expressed as

$$
v=\sum_{i=1}^{J} \lambda_{i} u_{i}
$$

where $u_{i} \in U(\alpha, k, v)$ and $0 \leq \lambda_{i} \leq 1, \sum_{i=1}^{J} \lambda_{i}=1$.

Proof. First of all, what we have to prove is that $v \in T(\alpha, k)$ is in the convex hull of $U(\alpha, k, v)$. To show the statement, we proceed by induction.

Suppose $v \in T(\alpha, k)$. If $v$ is block $k$-sparse, $v$ itself is in $U(\alpha, k, v)$. Thus, assume that the assertion is true for all block $(s-1)$-sparse vectors $v(s-1 \geq k)$, then we show that the assertion is also true for any block $s$-sparse vectors $v$. For any block $s$-sparse vectors $v \in T(\alpha, k)$, (without loss of generality, suppose that $v$ is not block $(s-1)$-sparse, otherwise the result holds by assumption of block $(s-1)$-sparse), we have $\|v\|_{2, \infty} \leq \alpha,\|v\|_{2,1} \leq k \alpha$. Furthermore, $v$ can be expressed as $v=\sum_{i=1}^{s} c_{i} E_{i}$ with $c_{1} \geq c_{2} \cdots \geq c_{s}>0$, where $c_{1}$ equals to the largest $\|v[i]\|_{2}$ for every $i \in\{1,2, \ldots, M\}, c_{2}$ equals to the next largest $\|v[i]\|_{2}$, and so on, where $E_{i}$ is a unit vector in $\mathbb{R}^{N}$, which equals to $v / c_{i}$ on the $i$ th largest block of $v$ and zero elsewhere. Let

$$
\Omega=\left\{1 \leq l \leq s-1: c_{l}+c_{l+1}+\cdots+c_{s} \leq(s-l) \alpha\right\} .
$$

Owing to $\sum_{i=1}^{s} c_{i}=\|v\|_{2,1} \leq k \alpha, \Omega$ is not empty for $1 \in \Omega$. We denote by $l$ the largest element in $\Omega$. It is easy to get

$$
\begin{aligned}
& c_{l}+c_{l+1}+\cdots+c_{s} \leq(s-l) \alpha, \\
& c_{l+1}+c_{l+2}+\cdots+c_{s}>(s-l-1) \alpha .
\end{aligned}
$$

It is worthy of noting that (2.1) also holds when the largest element in $\Omega$ is $s-1$. Take

$$
b_{j}=\frac{\sum_{i=l}^{s} c_{i}}{s-l}-c_{j}, \quad l \leq j \leq s .
$$


By direct calculations, we have $(s-l) \sum_{i=l}^{s} b_{i}=\sum_{i=l}^{s} c_{i}$ and $b_{j} \geq b_{l}$ for all $l \leq j \leq s$. Moreover, for any $l \leq j \leq s$,

$$
\begin{array}{r}
b_{j} \geq b_{l}=\frac{\sum_{i=l+1}^{s} c_{i}-(s-l-1) c_{l}}{s-l} \\
\geq \frac{\sum_{i=l+1}^{s} c_{i}-(s-l-1) \alpha}{s-l}>0,
\end{array}
$$

where the second inequality follows from the fact that $\|v\|_{2, \infty} \leq \alpha$, the last inequality is a result of the second inequality in (2.1). Next, define

$$
\lambda_{j}=\frac{b_{j}}{\sum_{i=l}^{s} b_{i}}, \quad v_{j}=\sum_{i=1}^{l-1} c_{i} E_{i}+\left(\sum_{i=l}^{s} b_{i}\right) \sum_{i=l, i \neq j}^{s} E_{i} \in \mathbb{R}^{N}
$$

for every $l \leq j \leq s$, we have

$$
v=\sum_{j=l}^{s} \lambda_{j} v_{j}, \quad 0<\lambda_{j} \leq 1, \quad \sum_{j=l}^{s} \lambda_{j}=1, \quad \operatorname{supp}\left(v_{j}\right) \subseteq \operatorname{supp}(v) .
$$

In addition, using the fact that $(s-l) \sum_{i=l}^{s} b_{i}=\sum_{i=l}^{s} c_{i},\|v\|_{2, \infty} \leq \alpha$ and the first inequality in (2.1), we have

$$
\begin{aligned}
& \left\|v_{j}\right\|_{2,1}=\sum_{i=1}^{l-1} c_{i}+(s-l) \sum_{i=l}^{s} b_{i}=\sum_{i=1}^{l-1} c_{i}+\sum_{i=l}^{s} c_{i}=\|v\|_{2,1}, \\
& \left\|v_{j}\right\|_{2, \infty}=\max \left\{c_{1}, \ldots, c_{l-1}, \sum_{i=l}^{s} b_{i}\right\} \leq \max \left\{\alpha, \frac{\sum_{i=l}^{s} c_{i}}{s-l}\right\} \leq \alpha .
\end{aligned}
$$

Finally, since $v_{j}$ is block $(s-1)$-sparse, under the induction assumption, we have $v_{j}=$ $\sum_{i=1}^{J} \mu_{j, i} u_{j, i}$, where $u_{j, i}$ is block $k$-sparse, $\left\|u_{j, i}\right\|_{2,1}=\left\|v_{j}\right\|_{2,1}=\|v\|_{2,1},\left\|u_{j, i}\right\|_{2, \infty} \leq \alpha$ and $0 \leq \mu_{j, i} \leq 1, \sum_{i=1}^{J} \mu_{j, i}=1$. Hence, $v=\sum_{i=1}^{J} \sum_{j=l}^{s} \lambda_{j} \mu_{j, i} u_{j, i}$, which implies that the statement is true for $s$.

On the other hand, if $v$ is in the convex hull of $U(\alpha, k, v)$, then $v=\sum_{i=1}^{J} \lambda_{i} u_{i}, u_{i} \in U(\alpha, k, v)$ and $0 \leq \lambda_{i} \leq 1, \sum_{i=1}^{J} \lambda_{i}=1$. It follows immediately that

$$
\begin{aligned}
\|v\|_{2,1} & =\left\|\sum_{i=1}^{J} \lambda_{i} u_{i}\right\|_{2,1} \leq \sum_{i=1}^{J} \lambda_{i}\left\|u_{i}\right\|_{2,1} \leq \sum_{i=1}^{J} \lambda_{i}\left\|u_{i}\right\|_{2,0}\left\|u_{i}\right\|_{2, \infty} \leq k \alpha \\
\|v\|_{2, \infty} & =\left\|\sum_{i=1}^{J} \lambda_{i} u_{i}\right\|_{2, \infty} \leq \sum_{i=1}^{J} \lambda_{i}\left\|u_{i}\right\|_{2, \infty} \leq \alpha
\end{aligned}
$$

which completes the proof.

The following lemma is a useful elementary inequality, which will be used in proving our main results. 
Lemma 2.2 ([9], Lemma 5.3) Assume $m \geq k, a_{1} \geq a_{2} \geq \cdots \geq a_{m} \geq 0, \sum_{i=1}^{k} a_{i} \geq \sum_{i=k+1}^{m} a_{i}$. Then for all $\alpha \geq 1$,

$$
\sum_{j=k+1}^{m} a_{j}^{\alpha} \leq \sum_{i=1}^{k} a_{i}^{\alpha}
$$

More generally, assume $a_{1} \geq a_{2} \geq \cdots \geq a_{m} \geq 0, \lambda \geq 0$ and $\sum_{i=1}^{k} a_{i}+\lambda \geq \sum_{i=k+1}^{m} a_{i}$, then for all $\alpha \geq 1$,

$$
\sum_{j=k+1}^{m} a_{j}^{\alpha} \leq k\left(\sqrt[\alpha]{\frac{\sum_{i=1}^{k} a_{i}^{\alpha}}{k}}+\frac{\lambda}{k}\right)^{\alpha}
$$

From the definition of $h, \widehat{x}$ and $x$, we have the following lemma.

Lemma 2.3. For any $\Gamma \subset\{1,2, \ldots, M\}$, it holds that

$$
\left\|h\left[\Gamma^{c}\right]\right\|_{2,1} \leq\|h[\Gamma]\|_{2,1}+2\left\|x\left[\Gamma^{c}\right]\right\|_{2,1} .
$$

Proof. Recall that $h=\widehat{x}-x$. From the minimality of $\widehat{x}$, it follows that

$$
\|\widehat{x}\|_{2,1} \leq\|x\|_{2,1}=\|x[\Gamma]\|_{2,1}+\left\|x\left[\Gamma^{c}\right]\right\|_{2,1}
$$

By the reverse triangle inequalities of $\|\cdot\|_{2,1}$, we obtain

$$
\begin{aligned}
\|\widehat{x}\|_{2,1} & =\|x+h\|_{2,1}=\|x[\Gamma]+h[\Gamma]\|_{2,1}+\left\|x\left[\Gamma^{c}\right]+h\left[\Gamma^{c}\right]\right\|_{2,1} \\
& \geq\|x[\Gamma]\|_{2,1}-\|h[\Gamma]\|_{2,1}+\left\|h\left[\Gamma^{c}\right]\right\|_{2,1}-\left\|x\left[\Gamma^{c}\right]\right\|_{2,1} .
\end{aligned}
$$

The lemma follows from above inequalities immediately.

\section{Main Results}

With the preparations given in Section 2, we establish the main results in this section-a sharp high order block RIP condition for the robust recovery of arbitrary signals with block pattern. When the signal is block sparse, the sharp condition ensures the exact recovery and stable recovery in the noiseless case and in the noisy case, respectively. First, the following theorem provides a sufficient condition of recovery when $x$ is not block sparse and the observation is noisy.

Theorem 3.1. Suppose that $x \in \mathbb{R}^{N}$ is an arbitrary vector consistent with (1.1) and $\|z\|_{2} \leq \varepsilon$. If the measurement matrix $A$ satisfies the block RIP with $\delta_{t k}<\sqrt{\frac{t-1}{t}}$ for $t>1$, the solution $\widehat{x}$ to $(1.2)$ obeys

$$
\begin{aligned}
\|\widehat{x}-x\|_{2} \leq & \frac{2 \sqrt{2 t(t-1)\left(1+\delta_{t k}\right)}}{t\left(\sqrt{(t-1) / t}-\delta_{t k}\right)} \varepsilon \\
& +\left(\frac{\sqrt{2} \delta_{t k}+\sqrt{t\left(\sqrt{(t-1) / t}-\delta_{t k}\right) \delta_{t k}}}{t\left(\sqrt{(t-1) / t}-\delta_{t k}\right)}+1\right) \frac{2\left\|x\left[\mathcal{I}_{0}^{c}\right]\right\|_{2,1}}{\sqrt{k}} .
\end{aligned}
$$


Proof. First of all, suppose that $t k$ is an integer. Let $\widehat{x}=x+h$, where $\widehat{x}$ is a solution to $l_{2} / l_{1}$ minimization problem and $x$ is the original signal. From Lemma 2.3 and the definition of $\mathcal{I}_{0},\left\|h\left[\mathcal{I}_{0}^{c}\right]\right\|_{2,1} \leq\left\|h\left[\mathcal{I}_{0}\right]\right\|_{2,1}+2\left\|x\left[\mathcal{I}_{0}^{c}\right]\right\|_{2,1}$. We assume that $T_{0}$ is the block index set over the $k$ blocks with largest $l_{2}$ norm of $h$. Hence,

$$
\left\|h\left[T_{0}^{c}\right]\right\|_{2,1} \leq\left\|h\left[T_{0}\right]\right\|_{2,1}+2\left\|x\left[\mathcal{I}_{0}^{c}\right]\right\|_{2,1}
$$

Denote $r=\left(\left\|h\left[T_{0}\right]\right\|_{2,1}+2\left\|x\left[\mathcal{I}_{0}^{c}\right]\right\|_{2,1}\right) / k$. Next, we decompose $h\left[T_{0}^{c}\right]$ as $h\left[T_{0}^{c}\right]=h\left[T_{1}\right]+h\left[T_{2}\right]$, where $h\left[T_{1}\right]$ remains the blocks of $h\left[T_{0}^{c}\right]$ whose $l_{2}$ norms are greater than $\frac{r}{t-1}$ and 0 elsewhere, $h\left[T_{2}\right]$ retains the blocks of $h\left[T_{0}^{c}\right]$ whose $l_{2}$ norms are not more than $\frac{r}{t-1}$ and 0 otherwise. Combining above definitions and (3.2), we have

$$
\left\|h\left[T_{1}\right]\right\|_{2,1} \leq\left\|h\left[T_{0}^{c}\right]\right\|_{2,1} \leq k r .
$$

Denote $\left\|h\left[T_{1}\right]\right\|_{2,0}=l$. From the definition of $h\left[T_{1}\right]$, we get

$$
k r \geq\left\|h\left[T_{1}\right]\right\|_{2,1}=\sum_{i \in \operatorname{supp}\left[h\left[T_{1}\right]\right]}\left\|h\left[T_{1}\right][i]\right\|_{2}>\frac{l r}{t-1} .
$$

Namely, $l<k(t-1)$. Thus, it is clear that

$$
\begin{aligned}
\left\|h\left[T_{2}\right]\right\|_{2,1} & =\left\|h\left[T_{0}^{c}\right]\right\|_{2,1}-\left\|h\left[T_{1}\right]\right\|_{2,1} \\
& <k r-\frac{l r}{t-1}=(k(t-1)-l) \cdot \frac{r}{t-1}, \\
\left\|h\left[T_{2}\right]\right\|_{2, \infty} & \leq \frac{r}{t-1}
\end{aligned}
$$

and $\left\|h\left[T_{0}\right]+h\left[T_{1}\right]\right\|_{2,0}=k+l<t k$. From the definition of $\delta_{k}$,

$$
\begin{aligned}
\left\langle A\left(h\left[T_{0}\right]+h\left[T_{1}\right]\right), A h\right\rangle & \leq\left\|A\left(h\left[T_{0}\right]+h\left[T_{1}\right]\right)\right\|_{2}\|A h\|_{2} \\
& \leq \sqrt{1+\delta_{t k}}\left\|h\left[T_{0}\right]+h\left[T_{1}\right]\right\|_{2}\|A h\|_{2} .
\end{aligned}
$$

Due to

$$
\|A h\|_{2} \leq\|A \widehat{x}-A x\|_{2} \leq\|A \widehat{x}-y\|_{2}+\|y-A x\|_{2} \leq 2 \varepsilon,
$$

so (3.4) becomes

$$
\left\langle A\left(h\left[T_{0}\right]+h\left[T_{1}\right]\right), A h\right\rangle \leq \sqrt{1+\delta_{t k}}\left\|h\left[T_{0}\right]+h\left[T_{1}\right]\right\|_{2} \cdot(2 \varepsilon) .
$$

Using (3.3a) and Lemma 2.1, we have $h\left[T_{2}\right]=\sum_{i=1}^{J} \lambda_{i} u_{i}$, where $u_{i}$ is block $(k(t-1)-l)$-sparse, $\sum_{i=1}^{J} \lambda_{i}=1$ with $0 \leq \lambda_{i} \leq 1$, and $\operatorname{supp}\left(u_{i}\right) \subseteq \operatorname{supp}\left(h\left[T_{2}\right]\right),\left\|u_{i}\right\|_{2,1}=\left\|h\left[T_{2}\right]\right\|_{2,1},\left\|u_{i}\right\|_{2, \infty} \leq \frac{r}{t-1}$. Hence,

$$
\begin{gathered}
\quad\left\|u_{i}\right\|_{2}=\left\|u_{i}\right\|_{2,2} \leq \sqrt{\left\|u_{i}\right\|_{2,0}}\left\|u_{i}\right\|_{2, \infty} \\
\leq \sqrt{k(t-1)-l} \cdot \frac{r}{t-1} \leq \sqrt{\frac{k}{t-1}} r
\end{gathered}
$$

where the first inequality follows from the fact that for any block $k$-sparse vector $v$,

$$
\|v\|_{2,2}^{2}=\sum_{i}\|v[i]\|_{2}^{2} \leq k\|v\|_{2, \infty}^{2}
$$


Let $X=\left\|h\left[T_{0}\right]+h\left[T_{1}\right]\right\|_{2,2}, P=2\left\|x\left[\mathcal{I}_{0}^{c}\right]\right\|_{2,1} / \sqrt{k}$. Clearly,

$$
\begin{aligned}
\left\|u_{i}\right\|_{2} & \leq \sqrt{\frac{k}{t-1}} r=\sqrt{\frac{k}{t-1}} \cdot \frac{\left\|h\left[T_{0}\right]\right\|_{2,1}+2\left\|x\left[\mathcal{I}_{0}^{c}\right]\right\|_{2,1}}{k} \\
& \leq \frac{\left\|h\left[T_{0}\right]\right\|_{2,2}}{\sqrt{t-1}}+\frac{2\left\|x\left[\mathcal{I}_{0}^{c}\right]\right\|_{2,1}}{\sqrt{k(t-1)}} \leq \frac{\left\|h\left[T_{0}\right]+h\left[T_{1}\right]\right\|_{2,2}}{\sqrt{t-1}}+\frac{2\left\|x\left[\mathcal{I}_{0}^{c}\right]\right\|_{2,1}}{\sqrt{k(t-1)}} \\
& =\frac{X+P}{\sqrt{t-1}}
\end{aligned}
$$

where the second inequality follows from applying Cauchy-Schwarz to any block $k$-sparse vector $v,\|v\|_{2,1}=\sum_{i}\|v[i]\|_{2} \cdot 1 \leq \sqrt{k}\|v\|_{2,2}$. Take $\beta_{i}=h\left[T_{0}\right]+h\left[T_{1}\right]+\mu u_{i}$, where $0 \leq \mu \leq 1$. We observe that

$$
\begin{aligned}
\sum_{j=1}^{J} \lambda_{j} \beta_{j}-\frac{1}{2} \beta_{i} & =h\left[T_{0}\right]+h\left[T_{1}\right]+\mu h\left[T_{2}\right]-\frac{1}{2} \beta_{i} \\
& =\left(\frac{1}{2}-\mu\right)\left(h\left[T_{0}\right]+h\left[T_{1}\right]\right)+\mu h-\frac{\mu}{2} u_{i} .
\end{aligned}
$$

Moreover, $\beta_{i}$ and $\sum_{j=1}^{N} \lambda_{j} \beta_{j}-\frac{1}{2} \beta_{i}-\mu h$ are block $t k$-sparse, due to the facts that $h\left[T_{0}\right]$ is block $k$-sparse, $h\left[T_{1}\right]$ is block $l$-sparse, and $u_{i}$ is block $k(t-1)-l$-sparse. Note that the following identity (see (25) in [8]) holds

$$
\sum_{i=1}^{J} \lambda_{i}\left\|A\left(\sum_{j=1}^{J} \lambda_{j} \beta_{j}-\frac{1}{2} \beta_{i}\right)\right\|_{2}^{2}=\sum_{i=1}^{J} \frac{\lambda_{i}}{4}\left\|A \beta_{i}\right\|_{2}^{2} .
$$

We first bound the left hand side of (3.9). Substituting (3.8) into the left hand side of (3.9) and combining (3.6) and the definition of block RIP, we have the upper bound

$$
\begin{aligned}
& \sum_{i=1}^{J} \lambda_{i}\left\|A\left(\sum_{j=1}^{J} \lambda_{j} \beta_{j}-\frac{1}{2} \beta_{i}\right)\right\|_{2}^{2}=\sum_{i=1}^{J} \lambda_{i}\left\|A\left(\left(\frac{1}{2}-\mu\right)\left(h\left[T_{0}\right]+h\left[T_{1}\right]\right)+\mu h-\frac{\mu}{2} u_{i}\right)\right\|_{2}^{2} \\
= & \sum_{i=1}^{J} \lambda_{i}\left\|A\left(\left(\frac{1}{2}-\mu\right)\left(h\left[T_{0}\right]+h\left[T_{1}\right]\right)-\frac{\mu}{2} u_{i}\right)\right\|_{2}^{2} \\
& \quad+2\left\langle A\left(\left(\frac{1}{2}-\mu\right)\left(h\left[T_{0}\right]+h\left[T_{1}\right]\right)-\frac{\mu}{2} h\left[T_{2}\right]\right), \mu A h\right\rangle+\mu^{2}\|A h\|_{2}^{2} \\
= & \sum_{i=1}^{J} \lambda_{i}\left\|A\left(\left(\frac{1}{2}-\mu\right)\left(h\left[T_{0}\right]+h\left[T_{1}\right]\right)-\frac{\mu}{2} u_{i}\right)\right\|_{2}^{2}+\mu(1-\mu)\left\langle A\left(h\left[T_{0}\right]+h\left[T_{1}\right]\right), A h\right\rangle \\
\leq & \left(1+\delta_{t k}\right) \sum_{i=1}^{J} \lambda_{i}\left\|\left(\frac{1}{2}-\mu\right)\left(h\left[T_{0}\right]+h\left[T_{1}\right]\right)-\frac{\mu}{2} u_{i}\right\|_{2}^{2}+\mu(1-\mu) \sqrt{1+\delta_{t k}}\left\|h\left[T_{0}\right]+h\left[T_{1}\right]\right\|_{2} \cdot(2 \varepsilon) \\
= & \left(1+\delta_{t k}\right) \sum_{i=1}^{J} \lambda_{i}\left(\left(\frac{1}{2}-\mu\right)^{2}\left\|h\left[T_{0}\right]+h\left[T_{1}\right]\right\|_{2}^{2}+\frac{\mu^{2}}{4}\left\|u_{i}\right\|_{2}^{2}\right)+\mu(1-\mu) \sqrt{1+\delta_{t k}}\left\|h\left[T_{0}\right]+h\left[T_{1}\right]\right\|_{2} \cdot(2 \varepsilon) .
\end{aligned}
$$


On the other hand, using the expression of $\beta_{i}$, the block RIP results in the lower bound

$$
\begin{aligned}
\sum_{i=1}^{J} \frac{\lambda_{i}}{4}\left\|A \beta_{i}\right\|_{2}^{2} & =\sum_{i=1}^{J} \frac{\lambda_{i}}{4}\left\|A\left(h\left[T_{0}\right]+h\left[T_{1}\right]+\mu u_{i}\right)\right\|_{2}^{2} \\
& \geq \sum_{i=1}^{J} \frac{\lambda_{i}}{4}\left(1-\delta_{t k}\right)\left\|h\left[T_{0}\right]+h\left[T_{1}\right]+\mu u_{i}\right\|_{2}^{2} \\
& =\left(1-\delta_{t k}\right) \sum_{i=1}^{J} \frac{\lambda_{i}}{4}\left(\left\|h\left[T_{0}\right]+h\left[T_{1}\right]\right\|_{2}^{2}+\mu^{2}\left\|u_{i}\right\|_{2}^{2}\right) .
\end{aligned}
$$

Combining the above two inequalities, we have

$$
\begin{aligned}
& {\left[\left(\mu^{2}-\mu\right)+\left(\frac{1}{2}-\mu+\mu^{2}\right) \delta_{t k}\right]\left\|h\left[T_{0}\right]+h\left[T_{1}\right]\right\|_{2}^{2}} \\
& \quad+\mu(1-\mu) \sqrt{1+\delta_{t k}}\left\|h\left[T_{0}\right]+h\left[T_{1}\right]\right\|_{2} \cdot(2 \varepsilon)+\sum_{i=1}^{J} \lambda_{i} \frac{\delta_{t k}}{2} \mu^{2}\left\|u_{i}\right\|_{2}^{2} \geq 0 .
\end{aligned}
$$

From (3.7) and the expression of $X$ with the fact $\|\cdot\|_{2,2}=\|\cdot\|_{2}$, we obtain

$$
\begin{aligned}
& {\left[\left(\mu^{2}-\mu\right)+\left(\frac{1}{2}-\mu+\left(1+\frac{1}{2(t-1)}\right) \mu^{2}\right) \delta_{t k}\right] X^{2}} \\
& \quad+\left[2 \varepsilon \mu(1-\mu) \sqrt{1+\delta_{t k}}+\frac{\mu^{2} \delta_{t k} P}{t-1}\right] X+\frac{\mu^{2} P^{2} \delta_{t k}}{2(t-1)} \geq 0 .
\end{aligned}
$$

Taking $\mu=\sqrt{t(t-1)}-(t-1)$, we have

$$
\frac{\mu^{2}}{t-1}\left[-t\left(\sqrt{\frac{t-1}{t}}-\delta_{t k}\right) X^{2}+\left(2 \varepsilon \sqrt{t(t-1)\left(1+\delta_{t k}\right)}+P \delta_{t k}\right) X+\frac{P^{2} \delta_{t k}}{2}\right] \geq 0 .
$$

The condition $\delta_{t k}<\sqrt{(t-1) / t}$ ensures that the above inequality is a second-order inequality for $X$ and the quadratic coefficient is negative. Thus, we obtain

$$
\begin{aligned}
X \leq\{( & \left.2 \varepsilon \sqrt{t(t-1)\left(1+\delta_{t k}\right)}+P \delta_{t k}\right)+\left[\left(2 \varepsilon \sqrt{t(t-1)\left(1+\delta_{t k}\right)}+P \delta_{t k}\right)^{2}\right. \\
& \left.\left.+2 t\left(\sqrt{(t-1) / t}-\delta_{t k}\right) P^{2} \delta_{t k}\right]^{1 / 2}\right\} \cdot\left(2 t\left(\sqrt{(t-1) / t}-\delta_{t k}\right)\right)^{-1} \\
\leq & \frac{2 \sqrt{t(t-1)\left(1+\delta_{t k}\right)}}{t\left(\sqrt{(t-1) / t}-\delta_{t k}\right)} \varepsilon+\frac{2 \delta_{t k}+\sqrt{2 t\left(\sqrt{(t-1) / t}-\delta_{t k}\right) \delta_{t k}}}{2 t\left(\sqrt{(t-1) / t}-\delta_{t k}\right)} P
\end{aligned}
$$

where the last inequality is a result of the fact that $(a+b)^{\frac{1}{2}} \leq a^{\frac{1}{2}}+b^{\frac{1}{2}}$ for any nonnegative constants $a$ and $b$. With (3.2) and the representation of $P$, it is clear that

$$
\left\|h\left[T_{0}^{c}\right]\right\|_{2,1} \leq\left\|h\left[T_{0}\right]\right\|_{2,1}+P \sqrt{k}
$$

From Lemma 2.2, we conclude that

$$
\left\|h\left[T_{0}^{c}\right]\right\|_{2,2} \leq\left\|h\left[T_{0}\right]\right\|_{2,2}+P
$$


Therefore, it is not hard to see that

$$
\begin{aligned}
\|h\|_{2}= & \sqrt{\left\|h\left[T_{0}\right]\right\|_{2}^{2}+\left\|h\left[T_{0}^{c}\right]\right\|_{2}^{2}} \leq \sqrt{\left\|h\left[T_{0}\right]\right\|_{2}^{2}+\left(\left\|h\left[T_{0}\right]\right\|_{2}+P\right)^{2}} \\
\leq & \sqrt{2}\left\|h\left[T_{0}\right]\right\|_{2}+P \leq \sqrt{2}\left(\left\|h\left[T_{0}\right]+h\left[T_{1}\right]\right\|_{2}\right)+P=\sqrt{2} X+P \\
\leq & \frac{2 \sqrt{2 t(t-1)\left(1+\delta_{t k}\right)}}{t\left(\sqrt{(t-1) / t}-\delta_{t k}\right)} \varepsilon \\
& +\left(\frac{\sqrt{2} \delta_{t k}+\sqrt{t\left(\sqrt{(t-1) / t}-\delta_{t k}\right) \delta_{t k}}}{t\left(\sqrt{(t-1) / t}-\delta_{t k}\right)}+1\right) \frac{2\left\|x\left[\mathcal{I}_{0}^{c}\right]\right\|_{2,1}}{\sqrt{k}} .
\end{aligned}
$$

If $t k$ is not an integer, we denote $t^{\prime}=\lceil t k\rceil / k$, then $t^{\prime} k$ is an integer and $t<t^{\prime}$. So we have $\delta_{t^{\prime} k}=\delta_{t k}<\sqrt{\frac{t-1}{t}}<\sqrt{\frac{t^{\prime}-1}{t^{\prime}}}$. Using the method similar to the proof above, we can prove the result by working on $\delta_{t^{\prime} k}$. Hence, we complete the proof of the theorem.

Remark 3.1. Recently, Zhang and Li in [49] have shown that when $0<t<\frac{4}{3}$, the condition $\delta_{t k}<\frac{t}{4-t}$ is sufficient to guarantee the exact recovery for all $k$-sparse signals in the noiseless case via the constrained $l_{1}$-norm minimization. These bounds are sharp as in the following theorem. These results give a complete answer to the conjecture on restricted isometry property constants $\delta_{t k}\left(0<t<\frac{4}{3}\right.$ which was proposed by Cai and Zhang in [8]. We will establish analogous block restricted isometry conditions for the exact and stable recovery of signals with block pattern by mixed $l_{2} / l_{1}$ minimization. Due to the big difference as in [8] and [49], we postpone the details to a separate paper.

Remark 3.2. Theorem 3.1 and Remark 3.1 indicate that as long as measurement matrix $A$ meets with the block RIP with a suitable constant, the mixed $l_{2} / l_{1}$ minimization method can robustly recover any signals with block structure from noisy measurements $y=A x+z$. Moreover, if $x$ is block $k$-sparse, then Theorem 3.1 guarantees perfect and stable recovery of $x$ from its samples $y$ in the noise-free and noisy setting.

The following theorem shows the condition $\delta_{t k}<\sqrt{\frac{t-1}{t}}$ with $t \geq \frac{4}{3}$ is sharp for exact and stable recovery in noiseless and noisy case, respectively.

Theorem 3.2. Let $t \geq \frac{4}{3}$. For any $\varepsilon>0$ and $k \geq \frac{5}{\varepsilon}$, then there exist a sensing matrix $A \in \mathbb{R}^{n \times N}$ with $\delta_{t k}<\sqrt{\frac{t-1}{t}}+\varepsilon$ and some block $k$-sparse signal $x_{0}$ such that

(1) In the noiseless case, i.e., $y=A x_{0}$, the mixed $l_{2} / l_{1}$ minimization (1.2) can not exactly recover the block $k$-sparse signal $x_{0}$, i.e., $\widehat{x} \neq x_{0}$, where $\widehat{x}$ is the solution to (1.2).

(2) In the noise case, i.e., $y=A x_{0}+z$, the mixed $l_{2} / l_{1}$ minimization (1.2) can not stably recover the block $k$-sparse signal $x_{0}$, i.e., $\widehat{x} \nrightarrow x_{0}$ as $z \rightarrow 0$, where $\widehat{x}$ is the solution to $(1.2)$.

Proof. For all $\varepsilon>0$ and $k \geq \frac{5}{\varepsilon}$, let $a^{\prime}=((t-1)+\sqrt{t(t-1)}) k$ and $N \geq\left(k+a^{\prime}\right) d$. Since $t \geq \frac{4}{3}$, we have $a^{\prime} \geq k$. Suppose that $a$ is the largest integer strictly smaller than $a^{\prime}$, then $a<a^{\prime}$ 
and $a^{\prime}-a \leq 1$. Denote

$$
\gamma=\frac{1}{\sqrt{k d+\frac{a k^{2}}{a^{\prime 2}} d}}(\overbrace{k \text { blocks }}^{\underbrace{d}_{1, \ldots, 1}, \ldots, \overbrace{1, \ldots, 1}^{d}}, \underbrace{\overbrace{-\frac{k}{a^{\prime}}, \ldots,-\frac{k}{a^{\prime}}, \ldots,-\frac{k}{a^{\prime}}, \ldots,-\frac{k}{a^{\prime}}}^{d}}_{a \text { blocks }}, \mathbf{0}, \ldots, \mathbf{0}) \in \mathbb{R}^{N},
$$

where $k+a \leq M, \mathcal{I}=\left\{d_{1}=d, \ldots, d_{k+a}=d, d_{k+a+1}, \ldots, d_{M}\right\}$ and $\|\gamma\|_{2}=1$. Define the linear $\operatorname{map} A: \mathbb{R}^{N} \rightarrow \mathbb{R}^{N}$ by

$$
A x=\sqrt{1+\sqrt{\frac{t-1}{t}}}(x-\langle\gamma, x\rangle \gamma),
$$

for all $x \in \mathbb{R}^{N}$. Then for any block $\lceil t k\rceil$-sparse signal $x$, we have

$$
\|A x\|_{2}^{2}=\left(1+\sqrt{\frac{t-1}{t}}\right)\left(\|x\|_{2}^{2}-|\langle\gamma, x\rangle|^{2}\right) .
$$

From the Cauchy-Schwarz inequality and $a^{\prime} \geq k, a^{\prime}-a \leq 1$ as well as the fact $\frac{a^{\prime 2}+k^{2}(t-1)}{a^{\prime 2}+a^{\prime} k}=$ $2 \sqrt{t-1}(\sqrt{t}-\sqrt{t-1})$ (see the proof of Theorem 2.2, [8]), therefore,

$$
\begin{aligned}
0 & \leq|\langle\gamma, x\rangle|^{2}=|\langle\gamma[\operatorname{supp}[x]], x\rangle|^{2} \leq\|x\|_{2}^{2} \cdot\|\gamma[\max (\lceil t k\rceil)]\|_{2}^{2} \\
& \leq\|x\|_{2}^{2} \cdot \frac{a^{\prime 2}+k(\lceil t k\rceil-k)}{a^{\prime 2}+a k} \leq \frac{a^{\prime 2}+k^{2}(t-1)+k}{a^{\prime 2}+a k}\|x\|_{2}^{2} \\
& =\frac{a^{\prime 2}+k^{2}(t-1)+k}{a^{\prime 2}+a^{\prime} k} \cdot \frac{a^{\prime 2}+a^{\prime} k}{a^{\prime 2}+a k}\|x\|_{2}^{2} \\
& =\frac{a^{\prime 2}+k^{2}(t-1)+k}{a^{\prime 2}+a^{\prime} k} \cdot \frac{1}{1-\frac{k\left(a^{\prime}-a\right)}{a^{\prime 2}+a^{\prime} k}}\|x\|_{2}^{2} \\
& =\frac{a^{\prime 2}+k^{2}(t-1)}{a^{\prime 2}+a^{\prime} k} \cdot \frac{a^{\prime 2}+k^{2}(t-1)+k}{a^{\prime 2}+k^{2}(t-1)} \cdot \frac{1}{1-\frac{k\left(a^{\prime}-a\right)}{a^{\prime 2}+a^{\prime} k}}\|x\|_{2}^{2} \\
& \leq 2 \sqrt{t-1}(\sqrt{t}-\sqrt{t-1}) \cdot\left(1+\frac{1}{t k}\right) \cdot \frac{1}{1-\frac{1}{2 k}\|x\|_{2}^{2}} \\
& \leq 2(\sqrt{t(t-1)}-(t-1)) \cdot\left(1+\frac{5}{2 k}\right)\|x\|_{2}^{2} \\
& \leq\left(2 \sqrt{t(t-1)}-2(t-1)+\frac{5}{2 k}\right)\|x\|_{2}^{2}, \\
& =(t-1)
\end{aligned}
$$

where $\gamma[\max (\lceil t k\rceil)]$ denotes a vector remaining the $\lceil t k\rceil$ blocks with largest $l_{2}$ norm of $\gamma$ and zero elsewhere and $\|\gamma[\max (\lceil t k\rceil)]\|_{2}^{2} \leq \frac{a^{\prime 2}+k(\lceil t k\rceil-k)}{a^{\prime 2}+a k}$. The last inequality follows that $2 \sqrt{t(t-1)}-$ 
$2(t-1) \leq 1$. Consequently,

$$
\begin{aligned}
& \left(1+\sqrt{\frac{t-1}{t}}+\varepsilon\right)\|x\|_{2}^{2} \geq\left(1+\sqrt{\frac{t-1}{t}}\right)\|x\|_{2}^{2} \geq\|A x\|_{2}^{2} \\
\geq & \left(1+\sqrt{\frac{t-1}{t}}\right)\left(1-2 \sqrt{t(t-1)}+2(t-1)-\frac{5}{2 k}\right)\|x\|_{2}^{2} \\
= & {\left[\left(1+\sqrt{\frac{t-1}{t}}\right)(1-2 \sqrt{t(t-1)}+2(t-1))-\left(1+\sqrt{\frac{t-1}{t}}\right) \frac{5}{2 k}\right]\|x\|_{2}^{2} } \\
= & {\left[1-\sqrt{\frac{t-1}{t}}-\left(1+\sqrt{\frac{t-1}{t}}\right) \frac{5}{2 k}\right]\|x\|_{2}^{2} \geq\left(1-\sqrt{\frac{t-1}{t}}-\varepsilon\right)\|x\|_{2}^{2}, }
\end{aligned}
$$

where the last inequality follows from that $1+\sqrt{\frac{t-1}{t}} \leq 2$ and $k \geq \frac{5}{\varepsilon}$. It follows immediately that $\delta_{t k} \leq \sqrt{\frac{t-1}{t}}+\varepsilon$. Next, we define

$$
\begin{aligned}
& x_{0}=(\overbrace{k \text { blocks }}^{\overbrace{1, \ldots, 1}^{d}, \ldots, \overbrace{1, \ldots, 1}^{d}}, \underbrace{\overbrace{0, \ldots, 0}^{d}, \ldots, \overbrace{0, \ldots, 0}^{d}, \mathbf{0}, \ldots, \mathbf{0}) \in \mathbb{R}^{N},}_{\text {a blocks }} \\
& \gamma_{0}=(\overbrace{\underbrace{d, \ldots, 0}_{\text {blocks }}, \ldots, \overbrace{0, \ldots, 0}^{d}}^{\underbrace{d}}, \underbrace{\overbrace{\frac{k}{a^{\prime}}, \ldots, \frac{k}{a^{\prime}}}^{d}, \ldots, \overbrace{\frac{k}{a^{\prime}}, \ldots, \frac{k}{a^{\prime}}}^{d}}_{\text {a blocks }}, \mathbf{0}, \ldots, \mathbf{0}) \in \mathbb{R}^{N} .
\end{aligned}
$$

Note that $\left\|x_{0}\right\|_{2,1}=k \sqrt{d},\left\|\gamma_{0}\right\|_{2,1} \leq \frac{a}{a^{\prime}} \cdot k \sqrt{d}<k \sqrt{d}$ and $x_{0}$ is block $k$-sparse, $\gamma=\frac{1}{\sqrt{k d+\frac{a k^{2}}{a^{\prime 2}}}}$ $\left(x_{0}-\gamma_{0}\right)$. Since $A \gamma=0$, we have $A x_{0}=A \gamma_{0}$.

Thus, in the noiseless case $y=A x_{0}$, suppose that the mixed $l_{2} / l_{1}$ minimization method (1.2) can exactly recover $x_{0}$, i.e., $\widehat{x}=x_{0}$. From the definition of $\widehat{x}$ and $y=A \gamma_{0}$, it contradicts that $\left\|\gamma_{0}\right\|_{2,1}<\left\|x_{0}\right\|_{2,1}$.

In the noisy case $y=A x_{0}+z$, suppose that the mixed $l_{2} / l_{1}$ minimization method (1.2) can stably recover $x_{0}$, i.e., $\lim _{z \rightarrow 0} \widehat{x}=x_{0}$. We observe that $y-A\left(\widehat{x}-x_{0}+\gamma_{0}\right)=y-A \widehat{x}$, thus $\|\widehat{x}\|_{2,1} \leq\left\|\widehat{x}-x_{0}+\gamma_{0}\right\|_{2,1}$. So $\left\|x_{0}\right\|_{2,1} \leq\left\|\gamma_{0}\right\|_{2,1}$ as $z \rightarrow 0$. It contradicts that $\left\|\gamma_{0}\right\|_{2,1}<\left\|x_{0}\right\|_{2,1}$.

Therefore, the mixed $l_{2} / l_{1}$ minimization method (1.2) fails to exactly and stably recover $x_{0}$ based on $y$ and $A$.

\section{Conclusion}

In this paper, we consider the problem of recovering for an unknown signal with additional structure-its entries are not dispersing over all the signal vector but arising in clusters (or blocks)-from a given set of noisy linear measurements. Based on block RIP, we mainly investigate the recovery guarantee for the mixed $l_{2} / l_{1}$ minimization method. By extending the technique of sparse representation of a polytope to the block setting, we establish a high order block RIP condition for robust recovery of signals with block pattern by mixed $l_{2} / l_{1}$ minimization in the presence of noise. We also proved under the same condition, the block sparse signals 
can be exactly and stably recovered in the noiseless and noisy cases, respectively. Furthermore, in order to clarify its optimality, we also give a specific measurement matrix and block sparse signal such that the given concrete signal can not be exactly and stably recovered from its samples via mixed $l_{2} / l_{1}$ minimization, when the upper bound of $\delta_{t k}$ increases any $\varepsilon$. Also, if $d_{i}=1$ for $i \in\{1, \ldots, M\}$, our results return to those of Cai and Zhang [8].

Acknowledgments. The authors thank the referees for their valuable suggestion and comments. This work was supported by the NSF of China (Nos.11801509, 11871109).

\section{References}

[1] M. Akcakaya, V. Tarokh, A frame construction and a universal distortion bound for sparse representations, IEEE Trans. Signal Process., 56:6 (2008), 2443-2450.

[2] F.R. Bach, Consistency of the group Lasso and multiple kernel learning, J. Mach. Learn. Res., 9 (2008), 1179-1225.

[3] R. Baraniuk, M. Davenport, R. DeVore, M. Wakin, A simple proof of the restricted isometry property for random matrices, Constr. Approx., 28:3 (2008), 253-263.

[4] P. Bechler, P. Wojtaszczyk, Error estimates for orthogonal matching pursuit and random dictionaries, Constr. Approx., 33:2 (2011), 273-288.

[5] T.T. Cai, L. Wang, G.W. Xu, New bounds for restricted isometry constants, IEEE Trans. Inform. Theory, 56:9 (2010), 4388-4394.

[6] T.T. Cai, L. Wang, G.W. Xu, Shifting inequality and recovery of sparse signals, IEEE Trans. Signal Process., 58:3 (2010), 1300-1308.

[7] T.T. Cai, G.W. Xu, J. Zhang, On recovery of sparse signal via $l_{1}$ minimization, IEEE Trans. Inform. Theory, 55:7 (2009), 3388-3397.

[8] T.T. Cai, A. Zhang, Spares representation of a polytope and recovery of sparse signals and lowrank matrices, IEEE Trans. Inform. Theory, 60:1 (2014), 122-132.

[9] T.T. Cai, A. Zhang, Sharp RIP bound for sparse signal and low-rank matrix recovery, Appl. Comput. Harmon. Anal., 35 (2013), 74-93.

[10] T.T. Cai, A. Zhang, Compressed sensing and affine rank minimization under restricted isometry, IEEE Trans. Signal Process., 61:13 (2013), 3279-3290.

[11] E.J. Candès, The restricted isometry property and its implications for compressed sensing, Compte Rendus Math., 346:9-10 (2008), 589-592.

[12] E.J. Candès, J. Romberg, T. Tao, Stable signal recovery from incomplete and inaccurate measurements, Commum. Pure Appl. Math., 59 (2006), 1207-1223.

[13] E.J. Candès, T. Tao, Decoding by linear programming, IEEE Trans. Inform. Theory, 51:12 (2005), 4203-4215.

[14] C. Chesneau, M. Hebiri, Some theoretical results on the Grouped Variables Lasso, Math. Methods Statist., 17:4 (2008), 317-326.

[15] A. Cohen, W. Dahmen, R. DeVore, Comopressed sensing and best $k$-term approximation, J. Am. Math. Soc., 22:1 (2009), 211-231.

[16] A. Cohen, W. Dahmen, R. DeVore, Instance optimal decoding by thresholding in compressed sensing, Harmonic Analysis and Partial Differential Equations, 505 (2010), 1-28.

[17] A. Cohen, W. Dahmen, R. DeVore, Orthogonal matching pursuit under the restricted isometry property, Constr. Approx., 45:1 (2017), 113-127.

[18] S. Cotter, B. Rao, Sparse channel estimation via matching pursuit with application to equalization, IEEE Trans. Commun., 50:3 (2002), 374-377.

[19] Y.C. Eldar, P. Kuppinger, H. Bolcskei, Block-sparse signals: uncertainty relations and efficient recovery, IEEE Trans. Signal Process., 58:6 (2010), 3042-3054. 
[20] Y.C. Eldar, M. Mishali, Robust recovery of signals from a structured union of subspaces, IEEE Trans. Inform. Theory, 55:11 (2009), 5302-5316.

[21] Y.C. Eldar, H. Rauhut, Average case analysis of multichannel sparse recovery using convex relaxation, IEEE Trans. Inform. Theory, 56:1 (2010), 505-519.

[22] E. Elhamifar, R. Vidal, Block-sparse recovery via convex optimization, IEEE Trans. Signal Process., 60:8 (2012), 4094-4107.

[23] S. Foucart, A note on guaranteed sparse recovery via $l_{1}$-minimization, Appl. Comput. Harmon. Anal., 29 (2010), 97-103.

[24] S. Foucart, Sparse recovery algorithms: sufficient conditions in terms of restricted isometry constants, Springer Proceedings in Mathematics, 13 (2012), 65-77.

[25] Y.L. Fu, H. F. Li, Q.H. Zhang, J. Zou, Block-sparse recovery via redundant block OMP, Signal Process., 97 (2014), 162-171.

[26] M. Herman, T. Strohmer, High-resoluton radar via compressed sensing, IEEE Trans. Signal Process., 57:6 (2009), 2275-2284.

[27] J. Huang, T. Zhang, The benefit of group sparsity, Ann. Stat., 38:4 (2010), 1978-2004.

[28] J.H. Lin, S. Li, Block sparse recovery via mixed $l_{2} / l_{1}$ minimization, Acta Math. Sin., 29:7 (2013), 1401-1412.

[29] E. Liu, V.N. Temlyakov, The orthogonal super greedy algorithm and applications in compressed sensing, IEEE Trans. Inform. Theory, 58:4 (2012), 2040-2047.

[30] E.D. Livshitz, V.N. Temlyakov, Sparse approximation and recovery by greedy algorithms, IEEE Trans. Inform. Theory, 60:7 (2014), 3989-4000.

[31] M. Lustig, D.L. Donoho, J.M. Santos, J.M. Pauly, Compressed sensing MRI, IEEE Signal Process. Mag., 25:2 (2008), 72-82.

[32] X.L. Lv, C.R. Wan, G.A. Bi, Block orthogonal greedy algorithm for stable recovery of block-sparse signal representations, Signal Process., 90 (2010), 3265-3277.

[33] A. Majumdar, R.K. Ward, Compressen sensing of color images, Signal Process., 90:12 (2010), 3122-3127.

[34] L. Meier, S. Geer, P. Bühlmann, The group lasso for logistic regression, J. R. Statist. Soc. B, 70:1 (2008), 53-77.

[35] M. Mishali, Y.C. Eldar, Blind multi-band signal reconstruction: compressed sensing for analog signals, IEEE Trans. Signal Process., 57:3 (2009), 993-1009.

[36] M. Mishali, Y.C. Eldar, Reduce and boost: Recovering arbitrary sets of jointly sparse vectors, IEEE Trans. Signal Process., 56:10 (2008), 4692-4702.

[37] Q. Mo, S. Li, New bounds on the restricted isometry constant $\delta_{2 k}$, Appl. Comput. Harmon. Anal., 31:3 (2011), 460-468.

[38] Y. Nardi, A. Rinaldo, On the asymptotic properties of the group Lasso estimator for linear models, Electron. J. Statist., 2 (2008), 605-633.

[39] F. Parvaresh, H. Vikalo, S. Misra, B. Hassibi, Recovering sparse signals using sparse measurement matrices in compressed DNA microarrays, IEEE J. Sel. Top. Signal Process., 2:3 (2008), 275-285.

[40] H. Rauhut, Compressive sensing and structured random matrices, Radon, 9 (2010), 1-92.

[41] H. Rauhut, J. Romberg, J. Tropp, Restricted isometries for partial random circulant matrices, Appl. Comput. Harmom. Anal., 32:2 (2012), 242-254.

[42] V.N. Temlyakov, Greedy approximation in convex optimization, Constr. Approx., 41:2 (2015), 269-296.

[43] J.A. Tropp, Greed is good: algorithmic results for sparse approximation, IEEE Trans. Inform. Theory, 50:10 (2004), 2231-2242.

[44] J.A. Tropp, A. Gilbert, Signal recovery from partial information via orthogonal matching pursuit, IEEE Trans. Inform. Theory, 53:12 (2007), 4655-4666.

[45] L. Wang, G.A. Bi, C.R. Wan, X.L. Lv, Improved stability conditions of BOGA for noisy blocksparse signals, Signal Process., 91 (2011), 2567-2574. 
[46] Y. Wang, J.J. Wang, Z.B. Xu, Restricted $p$-isometry properties of nonconvex block-sparse compressed sensing, Signal Process., 104 (2014), 188-196.

[47] J.M. Wen, Z.C. Zhou, Z.L. Liu, M.J. Lai, X.H. Tang, Sharp sufficient conditions for stable recovery of block sparse signals by block orthogonal matching pursuit, arXiv:1605.02894v1 [cs.IT] (May 2016).

[48] M. Yuan, Y. Lin, Model selection and estimation in regression with grouped variables, J. $R$. Statist. Soc. B, 68:1 (2006), 49-67.

[49] R. Zhang, S. Li, A proof of conjecture on restricted isometry property constants $\delta_{t k}(0<t<4 / 3)$, IEEE Trans. Inform. Theory, 64 (2018), 1699-1705.

[50] J. Zhang, D. Zhu, G. Zhang, Adaptive compressed sensing radar oriented toward cognitive detection in dynamic sparse target scene, IEEE Trans. Signal Process., 60:4 (2012), 1718-1729. 